\title{
ON SOME SMALL IANTHINA FANTHINA (L.) STRANDED ON THE ISLES OF SCILLY, 1957
}

\author{
By Douglas P. Wilson, D.Sc. \\ The Plymouth Laboratory
}

(Text-fig. I)

In an earlier paper (Wilson \& Wilson, I956) there was described a large-scale stranding, on the shores of Cornwall and Devon in 1954, of the pelagic gastropod Ianthina janthina (L.). Most of the shells then collected were broken, but thirty-six were entire and measurements of these showed that, in spite of considerable variation in the proportion of width to height, the width is proportionately greater in young than in old shells. None of the I954 shells was less than half an inch high, the majority being considerably higher. Two shells stranded at Sennen several years before, and the figure of one in a paper by Fowler (1947, as I. planispirata Adams \& Reeve) were the only specimens less than half-an-inch high available for comparison with the larger ones. The measurements of these agreed with the general conclusion that as the shell grows it becomes proportionately less wide. In the past these variations in shell shape have been responsible for the erection of a number of different species. In order to establish the conclusion firmly it was desirable to examine a collection of young shells.

On 3 I March 1957 Mrs M. Hicks wrote from St Agnes, Isles of Scilly, to say that on that day her husband had found eight small Ianthina, six of which had stranded alive on the afternoon's tide, the other two, one of them alive, earlier. Subsequently more were collected. On 8 April Miss Deborah Hicks (age 9) picked up about 130 , some of them living, some apparently not. On Io April about thirty-two more in the same general condition were obtained, and on II April another twenty-four, all apparently dead.

When examined in Plymouth all these specimens proved to be small I. janthina (L.), mostly of a shape formerly described as I. planispirata Adams \& Reeve. Almost all were less than half an inch high, being generally much smaller, and most of the shells were unbroken. Such a large collection of small shells of this species probably does not exist elsewhere, and it is therefore of interest to record their measurements and to compare them with the lesser collection of large shells described last year.

As before, the shells were measured with calipers graduated in hundredths of an inch. These measurements are recorded in Table I; the width/height ratios were again worked out. In the graph in Fig. I the ratio for each shell is 
TABLE 1. MEASUREMENT OF SHELLS OF IANTHINA IANTHINA (L.)

\begin{tabular}{|c|c|c|c|c|c|c|c|c|c|}
\hline $\begin{array}{l}\text { No. of } \\
\text { shells }\end{array}$ & $\begin{array}{l}\text { Totals: } \\
\text { each } \\
\text { height }\end{array}$ & $\begin{array}{l}\text { Height } \\
\text { (in.) }\end{array}$ & $\begin{array}{l}\text { Width } \\
\text { (in.) }\end{array}$ & $\begin{array}{l}\text { Ratio: } \\
\text { width } \\
\text { height }\end{array}$ & $\begin{array}{l}\text { No. of } \\
\text { shells }\end{array}$ & $\begin{array}{l}\text { Totals: } \\
\text { each } \\
\text { height }\end{array}$ & $\begin{array}{c}\text { Height } \\
\text { (in.) }\end{array}$ & $\begin{array}{l}\text { Width } \\
\text { (in.) }\end{array}$ & $\begin{array}{l}\text { Ratio: } \\
\text { width } \\
\text { height }\end{array}$ \\
\hline I & I & 0.22 & 0.28 & $\mathbf{I} \cdot 27$ & $\begin{array}{l}2 \\
2\end{array}$ & \multirow[t]{4}{*}{8} & $\begin{array}{l}0.34 \\
0.34\end{array}$ & $\begin{array}{l}0.40 \\
0.43\end{array}$ & $\begin{array}{l}\mathrm{I} \cdot \mathrm{I} 8 \\
\mathrm{I} \cdot 26\end{array}$ \\
\hline 2 & \multirow[t]{3}{*}{5} & 0.23 & 0.30 & $\mathrm{I} \cdot 30$ & I & & $\begin{array}{l}34 \\
0.34\end{array}$ & $\begin{array}{l}0.43 \\
0.44\end{array}$ & $\mathrm{I} \cdot 29$ \\
\hline 2 & & 0.23 & $0.3 \mathrm{I}$ & $\mathrm{I} \cdot 35$ & 2 & & 0.34 & 0.45 & $1 \cdot 32$ \\
\hline I & & 0.23 & 0.32 & I.39 & I & & 0.34 & 0.46 & $\mathrm{I} \cdot 35$ \\
\hline I & \multirow[t]{3}{*}{5} & 0.24 & $\begin{array}{l}0.31 \\
0.32\end{array}$ & $\begin{array}{r}1.29 \\
.28\end{array}$ & 2 & \multirow[t]{3}{*}{6} & 0.35 & 0.44 & $\begin{array}{l}I \cdot 26 \\
I \cdot 20\end{array}$ \\
\hline I & & $\begin{array}{l}0.24 \\
0.24\end{array}$ & $\begin{array}{l}0.33 \\
0.34\end{array}$ & $\begin{array}{l}\mathrm{I} \cdot 38 \\
\mathrm{I} \cdot 42\end{array}$ & $\begin{array}{l}\text { I } \\
3\end{array}$ & & $\begin{array}{l}0.35 \\
0.35\end{array}$ & $\begin{array}{l}0.45 \\
0.46\end{array}$ & $\begin{array}{l}\mathrm{r} \cdot 29 \\
\mathrm{r} 3 \mathrm{I}\end{array}$ \\
\hline 3 & & & & & & & & & \\
\hline I & \multirow[t]{2}{*}{4} & $\begin{array}{l}0.25 \\
0.25\end{array}$ & $\begin{array}{l}0.33 \\
0.34\end{array}$ & $\begin{array}{l}\mathrm{I} \cdot 32 \\
\mathrm{I} \cdot 36\end{array}$ & $\begin{array}{l}2 \\
3\end{array}$ & \multirow[t]{2}{*}{5} & $\begin{array}{l}0.36 \\
0.36\end{array}$ & \multirow{2}{*}{$\begin{array}{l}0.45 \\
0.47\end{array}$} & $\begin{array}{l}I .25 \\
I .2 I\end{array}$ \\
\hline $\begin{array}{l}2 \\
1\end{array}$ & & 0.25 & $\begin{array}{l}0.34 \\
0.37\end{array}$ & $\begin{array}{l}1 \cdot 36 \\
1 \cdot 48\end{array}$ & 3 & & 0.36 & & $I \cdot 3 I$ \\
\hline I & \multirow{5}{*}{5} & & & & I & \multirow[t]{5}{*}{6} & \multirow{5}{*}{$\begin{array}{l}0.37 \\
0.37 \\
0.37 \\
0.37 \\
0.37\end{array}$} & \multirow{5}{*}{$\begin{array}{l}0.46 \\
0.47 \\
0.48 \\
0.49 \\
0.50\end{array}$} & I. 24 \\
\hline I & & $\begin{array}{l}0.26 \\
0.26\end{array}$ & 0.34 & $I \cdot 3 I$ & I & & & & $1 \cdot 27$ \\
\hline I & & $\begin{array}{l}0.26 \\
0.26\end{array}$ & 0.35 & $\mathrm{I} \cdot 35$ & 2 & & & & $\mathrm{I} \cdot 30$ \\
\hline I & & $\begin{array}{l}0.26 \\
0.26\end{array}$ & 0.36 & I.38 & I & & & & $\begin{array}{r}\mathrm{I} \cdot 32 \\
\mathrm{I} \cdot 25\end{array}$ \\
\hline 2 & & 0.26 & 0.37 & $r \cdot 42$ & I & & & & $\mathrm{I} \cdot 35$ \\
\hline 3 & \multirow[t]{5}{*}{13} & 0.27 & 0.33 & $I \cdot 22$ & $\begin{array}{l}2 \\
I\end{array}$ & \multirow[t]{2}{*}{4} & \multirow{2}{*}{$\begin{array}{l}0.38 \\
0.38 \\
0.38\end{array}$} & \multirow{2}{*}{$\begin{array}{l}0.47 \\
0.48 \\
0.49\end{array}$} & $\begin{array}{l}I \cdot 24 \\
I \cdot 26\end{array}$ \\
\hline I & & $\begin{array}{l}0.27 \\
0.27\end{array}$ & 0.35 & $1 \cdot 30$ & $\begin{array}{l}1 \\
\text { I }\end{array}$ & & & & $\begin{array}{l}1 \cdot 26 \\
1 \cdot 29\end{array}$ \\
\hline $\begin{array}{l}I \\
4\end{array}$ & & 0.27 & $\begin{array}{l}0.36 \\
0.37\end{array}$ & $\begin{array}{l}\mathrm{I} \cdot 33 \\
\mathrm{I} \cdot 37\end{array}$ & I & I & 0.39 & $0.5 \mathrm{I}$ & $\mathrm{I} \cdot 3 \mathrm{I}$ \\
\hline 2 & & 0.27 & 0.38 & $\begin{array}{l}1.47 \\
\text { I } 4\end{array}$ & 1 & 0 & 0.40 & \multirow{2}{*}{-} & \multirow{2}{*}{-} \\
\hline 2 & & 0.27 & 0.39 & I. 44 & 0 & 0 & \multirow{2}{*}{$\begin{array}{l}0.40 \\
0.4 I\end{array}$} & & \\
\hline 3 & \multirow[t]{5}{*}{8} & 0.28 & 0.36 & I.29 & 1 & \multirow[t]{4}{*}{5} & & 0.52 & I.27 \\
\hline I & & 0.28 & 0.37 & $1 \cdot 32$ & I & & & 0.53 & $\begin{array}{l}\text { I.29 } \\
\end{array}$ \\
\hline 2 & & 0.28 & 0.38 & $I \cdot 36$ & I & & $\begin{array}{l}0.41 \\
0.4 \mathrm{I}\end{array}$ & $\begin{array}{l}0.54 \\
0.55\end{array}$ & $\begin{array}{l}\mathrm{I} \cdot 32 \\
\mathrm{I} \cdot 34\end{array}$ \\
\hline I & & 0.28 & 0.39 & I. 39 & I & & $0.4 \mathrm{I}$ & 0.57 & $1 \cdot 39$ \\
\hline I & & & 0.40 & I.43 & I & 3 & 0.42 & 0.54 & $\mathrm{I} \cdot 29$ \\
\hline I & I0 & 0.29 & 0.35 & $I \cdot 2 I$ & 2 & & 0.42 & 0.57 & $I \cdot 36$ \\
\hline 2 & & $\begin{array}{l}0.29 \\
0.29\end{array}$ & $\begin{array}{l}0.36 \\
0.27\end{array}$ & $\begin{array}{l}\text { I.24 } \\
\text { I.27 }\end{array}$ & 2 & 3 & 0.43 & 0.55 & I. 28 \\
\hline $\begin{array}{l}2 \\
1\end{array}$ & & $\begin{array}{l}0.29 \\
0.29\end{array}$ & $\begin{array}{l}0.37 \\
0.38\end{array}$ & & I & & 0.43 & 0.59 & $I \cdot 37$ \\
\hline I & & 0.29 & 0.39 & $\mathrm{I} \cdot 34$ & I & 3 & 0.44 & 0.56 & I. 27 \\
\hline 2 & & 0.29 & 0.40 & $1 \cdot 38$ & I & & 0.44 & 0.57 & $I \cdot 30$ \\
\hline I & & 0.29 & $0.4 \mathrm{I}$ & $\mathrm{I} \cdot 4 \mathrm{I}$ & I & & 0.44 & 0.58 & $\mathrm{I} \cdot 32$ \\
\hline 2 & I3 & 0.30 & 0.38 & $\mathbf{I} \cdot 27$ & I & 7 & 0.45 & 0.54 & $I \cdot 20$ \\
\hline 5 & & 0.30 & 0.39 & $1 \cdot 30$ & I & & 0.45 & 0.55 & $I \cdot 22$ \\
\hline 3 & & 0.30 & 0.40 & $\mathrm{I} \cdot 33$ & 2 & & 0.45 & 0.56 & I. 24 \\
\hline 2 & & 0.30 & $0.4 \mathrm{I}$ & $\mathbf{I} \cdot 37$ & 2 & & 0.45 & 0.60 & I.33 \\
\hline I & & 0.30 & 0.42 & I. 40 & I & & & & I. 38 \\
\hline I & 12 & 0.31 & 0.38 & $I \cdot 23$ & $\begin{array}{l}\text { I } \\
2\end{array}$ & 5 & 0.46 & 0.58 & $\begin{array}{l}\mathrm{I} \cdot 26 \\
\mathrm{I} \cdot 28\end{array}$ \\
\hline $\begin{array}{l}5 \\
3\end{array}$ & & 0.31 & 0.40 & $\begin{array}{l}1 \cdot 29 \\
1 \cdot 22\end{array}$ & $\begin{array}{l}2 \\
1\end{array}$ & & $\begin{array}{l}0.40 \\
0.46\end{array}$ & $\begin{array}{l}0.59 \\
0.60\end{array}$ & $\begin{array}{l}1.28 \\
I \cdot 30\end{array}$ \\
\hline $\begin{array}{l}3 \\
2\end{array}$ & & $\begin{array}{l}0.31 \\
0.31\end{array}$ & $\begin{array}{l}0.41 \\
0.42\end{array}$ & $\begin{array}{l}1 \cdot 32 \\
1 \cdot 36\end{array}$ & I & 2 & 0.47 & 0.57 & $I \cdot 2 I$ \\
\hline I & & 0.31 & 0.43 & $\mathrm{I} \cdot 39$ & I & & 0.47 & 0.60 & $\mathrm{I} \cdot 28$ \\
\hline I & I5 & 0.32 & 0.40 & I.25 & I & I & 0.48 & 0.60 & $\mathrm{I} \cdot 25$ \\
\hline 3 & & 0.32 & 0.41 & $\begin{array}{r}1 \cdot 28 \\
\end{array}$ & I & I & 0.49 & 0.59 & $I \cdot 20$ \\
\hline 5 & & $\begin{array}{l}0.32 \\
0.32\end{array}$ & 0.42 & $\begin{array}{l}I \cdot 3 I \\
I \cdot 34\end{array}$ & 3 & 5 & 0.50 & 0.64 & $I \cdot 28$ \\
\hline $\begin{array}{l}4 \\
\mathrm{I}\end{array}$ & & $\begin{array}{l}0.32 \\
0.32\end{array}$ & $\begin{array}{l}0.43 \\
0.44\end{array}$ & $\begin{array}{l}1 \cdot 34 \\
\mathrm{I} \cdot 38\end{array}$ & I & & 0.50 & 0.65 & $I \cdot 30$ \\
\hline I & & 0.32 & 0.48 & 1.50 & I & & 0.50 & 0.66 & $I \cdot 32$ \\
\hline I & 7 & 0.33 & 0.40 & $I \cdot 2 I$ & I & 2 & $0.5 \mathrm{I}$ & 0.64 & $I \cdot 26$ \\
\hline 3 & & 0.33 & 0.42 & I. 27 & 1 & & 0.51 & 0.67 & $I \cdot 3 I$ \\
\hline & & & 0.43 & $1 \cdot 30$ & I & $\mathbf{I}$ & 0.52 & 0.69 & $1 \cdot 33$ \\
\hline
\end{tabular}


plotted against the height. This graph is identical with that in Wilson \& Wilson (1956, text-fig. 2) with these shells added. It will be seen that their positions on the graph are in good agreement with the line which was originally based on the fewer and mainly larger shells measured previously. The shells marked I and II have not only wide apertures but also spires flatter than most. The shells marked III have average spires but their apertures, and hence crosssections of the coil, are noticeably narrow compared with most others; this gives these shells a tall appearance.

It appears that the shells comprise two main size-groups. The first of these contains 128 shells ranging in height from 0.22 to 0.39 in. with a peak (fifteen shells) at 0.32 in. The second group contains thirty-seven shells ranging in height from $0.4 \mathrm{I}$ to $0.52 \mathrm{in}$. with a peak (seven shells) at $0.45 \mathrm{in}$. These two groups are obvious in the graph (Fig. I) on either side of height

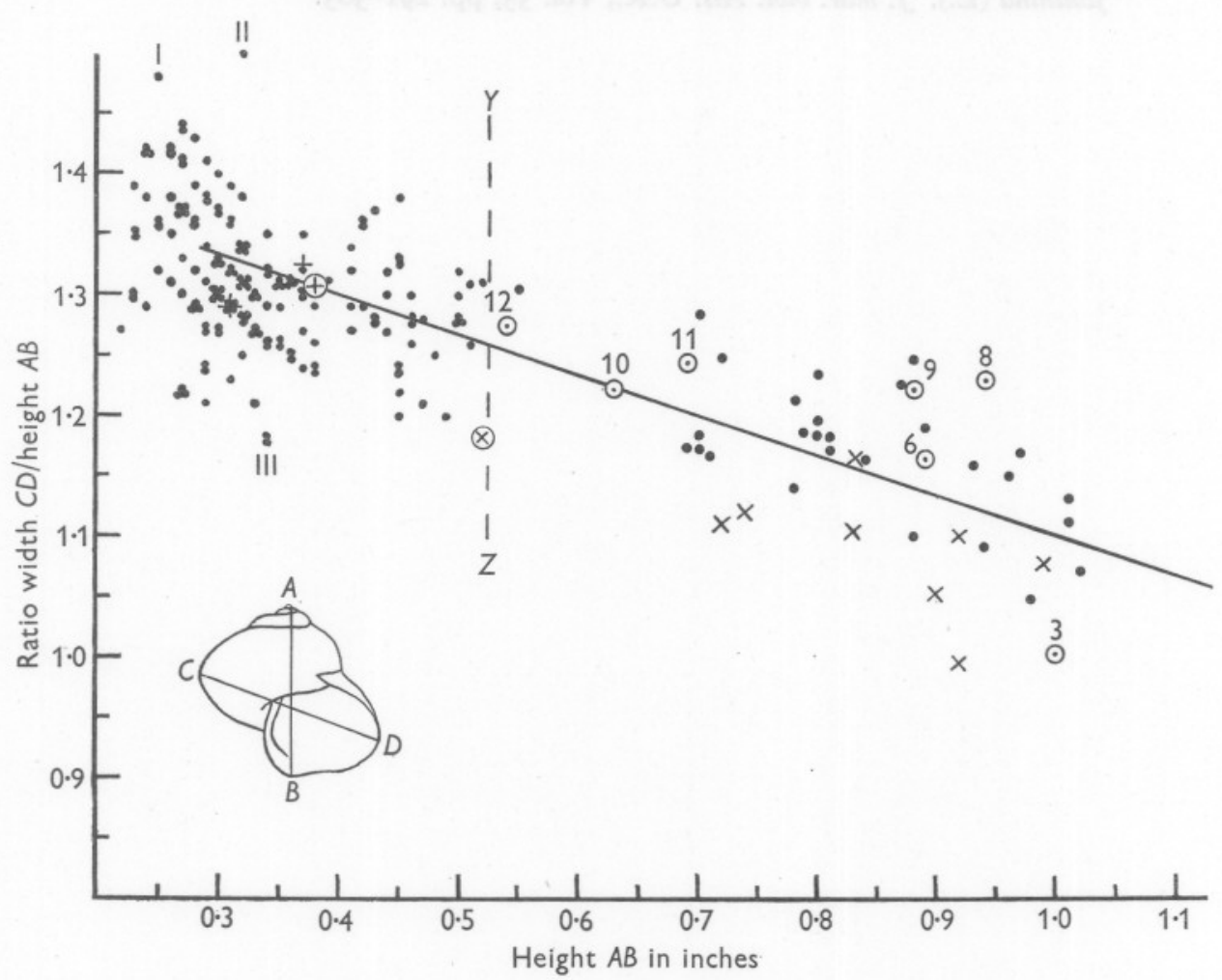

Fig. I. Left-hand portion of the graph reproduced in Wilson \& Wilson, 1956, p. 299, to which have been added, to the left of the broken line $Y Z$, data for young Ianthina janthina shells stranded in the Isles of Scilly, March and April 1957. The graph shows the relation of shell width to shell height, expressed as ratio of width $C D$ to height $A B$. Shells marked I-III are described in the text. For explanation of the remaining symbols see the legend to the original figure, reference as above. 
$0.40 \mathrm{in}$. There was no shell of that height in the collection. There is nothing to indicate what these groups represent, whether age, sex or other differences, but the presence of the peaks with almost regular falling off in numbers on either side (see Table I) makes it likely that they are not purely fortuitous.

The new shells add appreciably to the data previously available, especially for small sizes, and confirm earlier conclusions concerning the effect of growth on the relations between width and height. It only remains to express grateful thanks to $\mathrm{Mr}$ and Mrs Hicks, and especially their daughter Deborah, for collecting the shells and forwarding them with such care.

\section{REFERENCES}

FowleR, T. G. W., I947. Ianthina in Cornwall. F. Conch., Vol. 22, pt. II, p. I67. WILsON, D. P. \& WILson, M. A., I956. A contribution to the biology of Ianthina janthina (L.). F. mar. biol. Ass. U.K., Vol. 35, pp. 29I-305. 